\title{
TTR
}

Traduction, terminologie, rédaction

\section{Geneviève Roux-Faucard. Poétique du récit traduit. Paris, Lettres modernes Minard, 2008, 276 p.}

\section{Elisabeth Lortie}

Volume 24, numéro 2, 2e semestre 2011

URI : https://id.erudit.org/iderudit/1013406ar

DOI : https://doi.org/10.7202/1013406ar

Aller au sommaire du numéro

\section{Éditeur(s)}

Association canadienne de traductologie

ISSN

0835-8443 (imprimé)

1708-2188 (numérique)

Découvrir la revue

Citer ce compte rendu

Lortie, E. (2011). Compte rendu de [Geneviève Roux-Faucard. Poétique du récit traduit. Paris, Lettres modernes Minard, 2008, 276 p.] TTR, 24(2), 249-252.

https://doi.org/10.7202/1013406ar d'utilisation que vous pouvez consulter en ligne.

https://apropos.erudit.org/fr/usagers/politique-dutilisation/ 


\section{Geneviève Roux-Faucard. Poétique du récit traduit. Paris, Lettres modernes Minard, 2008, $276 \mathrm{p}$.}

Dans son livre, Geneviève Roux-Faucard jette un regard global, honnête et culturel sur la traduction du texte narratif littéraire. Pourquoi culturel? Parce qu'elle considère le processus traductif comme un changement d'arrière-plan culturel ou un dialogue culturel entre l'auteur, le traducteur et le lecteur. L'auteure fait une analyse exhaustive, sans toutefois donner de détails inutiles. Elle fait cette analyse en toute humilité parce qu'elle avoue ne pas avoir réponse à toutes les questions ne relevant pas nécessairement de son champ d'expertise ou directement du champ de son étude. Pour pallier la situation et afin que le lecteur puisse approfondir certaines notions à son gré, l'auteure fournit des références à consulter tout au long du texte et à la fin de l'ouvrage (près de six pages de références). Ce dernier est donc très bien documenté. Parmi les sujets qui peuvent être examinés en profondeur, on trouve notamment la narratologie comparée. L'auteure pose également des questions sur les relations entre la pensée et le langage : «Y $\mathrm{a}-\mathrm{t}-\mathrm{il}$ une teneur universelle du message qui s'exprime au moyen de la langue propre du locuteur ou la pensée de celui-ci est-elle conditionnée par sa langue? La langue est-elle simplement l'outil servant à dire une pensée déjà formée et qui la précède ou bien est-elle un espace clos à l'intérieur duquel toute pensée prend forme? " (p. 178). L'auteure suggère une piste de recherche en indiquant que le domaine du fonctionnement neurologique de la pensée et du langage serait probablement la clé.

Roux-Faucard effectue son étude de façon très rigoureuse : elle détermine la limite du corpus étudié; elle énonce, pratiquement au fur et à mesure quelles se soulèvent dans la tête du lecteur, les questions primordiales auxquelles elle tente de répondre; elle explique clairement les notions ainsi que les hypothèses avancées, qui peuvent sembler de prime abord complexes; elle montre les deux côtés de la médaille pour chacun des énoncés et cherche les points positifs et négatifs; elle fait des récapitulations et dresse des tableaux au moment opportun; elle isole les facteurs pour mieux les examiner. Finalement, elle cite de grands noms de la traductologie et de la critique littéraire, notamment Barthes, Genette, Eco, Bakhtine, Berman, 
Meschonnic et Riffaterre. Quant aux œuvres dans lesquelles Roux-Faucard puise ses exemples, on trouve entre autres $\mathrm{La}$ métamorphose (sur laquelle elle s'appuie tout au long de son livre) et Le château de Kafka, La fille du capitaine de Pouchkine, Amok de Sweig, L'Énéide de Virgile, Pays de neige de Kawabata, La femme des sables de Kōbō ainsi que Don Quichotte et Le mariage trompeur de Cervantès.

Le livre est très bien divisé : il est réparti en neuf courts chapitres, qui se lisent facilement et qui modulent adéquatement le raisonnement de l'auteure. Je n'ai d'ailleurs pas eu besoin de retourner en arrière pour reprendre la lecture d'un passage et il y a même un glossaire à la fin, au besoin. On y trouve des termes communs en traduction (adaptation, cibliste, correspondance, équivalence, révision, sourcière) ainsi que des termes plus spécialisés ou propres au champ d'études (p. ex., effet, lecteur possible, lecteur réel, littéralisante, modernisante, retraduction). Par contre, bien qu'elle souligne l'importance d'expliquer les termes étrangers, Roux-Faucard néglige de fournir, à plusieurs reprises, la traduction ou la signification de certains passages ou mots allemands, ce qui nuit à la compréhension des lecteurs qui ne maîtrisent pas l'allemand.

En ce qui concerne le contenu, le livre commence avec une citation de Barthes : "Innombrables sont les récits du monde » (p. 5), qui reflète parfaitement le ton du livre. En effet, si innombrables sont les récits du monde, innombrables sont les traductions, les stratégies et les analyses. Toujours à l'instar de Barthes, l'auteure donne sa place au lecteur (qui est également le traducteur). Elle parle même de l'aporie du traducteur, qui n'est pas sans rappeler la mort de l'auteur de Barthes. De plus, l'auteure se penche sur le fait que la tâche des traducteurs n'est pas de traduire une langue, mais bien un auteur. Elle traite aussi du comportement du texte traduit dans son nouvel environnement et du vieillissement des traductions, ce qui nécessite donc des retraductions au fil du temps. Elle relève également les différentes stratégies traductives, leurs aspects négatifs et positifs, les problèmes qu'elles engendrent ou les questions qu'elles soulèvent ainsi que les conséquences qu'elles entraînent sur le texte traduit, sa durée de vie et sa qualité. En ce qui concerne la « qualité » d'une traduction, Roux-Faucard établit la distinction entre la traduction 
initiale d'un auteur dans une langue, les premières traductions des œuvres de cet auteur et les retraductions subséquentes. La traduction idéale d'une œuvre s'obtient par des étapes de traduction, chacune ajoutant une « couche » à la qualité. C'est un concept qu'elle élaborera tout au long de son livre; elle consacre d'ailleurs un chapitre à la justification de ce concept qui veut que les retraductions soient de meilleure qualité que les traductions initiales ou les premières traductions. L'auteure traite également des notions d'intertextualité, d'épitextualité, d'intratextualité et de palimpseste ainsi que des polémiques qui règnent dans le monde de la traduction depuis longtemps, comme la polémique au sujet de la traduction littérale, de l'impossibilité de la traduction et de l'éthique du traducteur.

Je voudrais nuancer mes propos. Premièrement, comme les éléments de l'analyse ne s'appliquent pas à tous les types de récit, l'auteure s'est appuyée sur des romans qui se situent dans une petite catégorie du corpus littéraire (c'est-à-dire des œuvres dignes d'au moins deux traductions en français, pour le bien de son étude) puisque c'est grâce à de tels romans qu'il est possible d'effectuer les meilleures analyses. Par contre, il aurait été intéressant qu'elle fasse tout de même un parallèle avec la " littérature de consommation courante ", qu'elle a mise de côté. Deuxièmement, elle mentionne, au chapitre 1 , que "rien n'interdit au lecteur de faire du récit une autre lecture que celle que le texte préconise » (p. 18). C'est un point extrêmement important à mes yeux et qui n'est cependant pas suffisamment pris en considération au fil du livre.

En définitive, Poétique du récit traduit est un excellent livre, qui dresse un portrait juste et complet de la traduction des textes narratifs littéraires. L'auteure est objective, quoique son jugement soit teinté des idées des auteurs qu'elle cite, notamment Barthes. Elle réussit de main de maître à intégrer un nombre important de notions et à établir les liens entre elles, sans perdre de vue le sujet principal. Roux-Faucard conclut très bien son livre : «Traduire le texte narratif, lire le texte narratif traduit, c'est à la fois rencontrer ce qui est commun aux hommes de toutes les cultures et affronter ce qui est autre; accepter ce qui est à l'autre en le confrontant au propre » (p. 256). Dans son analyse, elle ne parle jamais d'erreur, 
d'infidélité ou de faute. Et elle a raison : toute traduction et toute retraduction contribuent à l'évolution de l'œuvre.

\author{
Elisabeth Lortie \\ UNIVERSITÉ D'OTTAWA
}

\title{
Maria Tymoczko. Enlarging Translation, Empowering Translators. St. Jerome, Manchester, 2007, 353 p.
}

In the rhetoric of self-congratulation in Translation Studies, civil engineering provides a rich source of metaphor. Translators are always building bridges, opening up channels and laying foundations. And of course there are rogues on the construction site who ignore the health and safety regulations (ethics) and leave Babel with dodgy tower blocks, monumentalizing their own bad faith. Maria Tymoczko, in this important book, expresses her impatience with these complacent, irenic definitions of translation which naturalise specific Western historical experiences and universalize them as binding descriptions of what translation is and is not.

The four chapters of Part 1 consider the implications of enlarging conventional understandings of translation practice and product, while the following four chapters in Part 2 investigate the notion of the empowerment of translators. The opening chapter sketches out an alternative reading of translation in the post-war period and chapter two addresses the core issue of how translation might be defined. In chapter three, Tymoczko explores the categories of representation, transmission and transculturation and analyzes their significance for the understanding of translation, and chapter four demonstrates how a broader understanding of the nature of translation would inform different research practices. Chapter five pays particular attention to the operations of translators in post-colonial societies as a means of engaging with ideas of empowerment, and chapter six looks at how holistic approaches to translation open up new perspectives for understanding the agency of the translator. Chapter seven takes on the central importance of theories of meaning for translation, while chapter eight investigates what ethics itself might mean for newly empowered translators. 